\title{
New Higgs signals induced by mirror fermion mixing effects
}

\author{
U. Cotti ${ }^{1}$. J. L. Diaz-Cruz ${ }^{2}$, R. Gaitán ${ }^{3}$, H H. Gonzales ${ }^{4}$, and A. Hernández-Galeana ${ }^{5}$ \\ ${ }^{1}$ IFM-UMSNH, A.P. 2-82, 58041, Morelia, Mich. México. \\ ${ }^{2}$ Instituto de Física, BUAP, A.P. J-48, 72570 Puebla, Pue. México \\ ${ }^{3}$ Centro de Investigaciones Teóricas, Facultad de Estudios Superiores-Cuautitlan, \\ A.P. 142, Cuautitlán-Izcalli, Estado de México, C. P. 54770, México. \\ ${ }^{4}$ Departamento de Física, Universidad Surcolombiana, A. A. 385, Neiva, Colombia. \\ ${ }^{5}$ Departamento de Física, Escuela Superior de Física y Matemáticas, \\ Instituto Politécnico Nacional, U.P. Adolfo López Mateos, C. P. 07r738, México.
}

(Dated: October 28, 2018)

\begin{abstract}
We study the conditions under which flavor violation arises in scalar-fermion interactions, as a result of the mixing phenomena between the standard model and exotic fermions. Phenomenological consequences are discussed within the specific context of a left-right model where these additional fermions have mirror properties under the new $S U(2)_{R}$ gauge group. Bounds on the parameters of the model are obtained from LFV processes; these results are then used to study the LFV Higgs decays $\left(H \rightarrow \tau l_{j}, l_{j}=e, \mu\right)$, which reach branching ratios that could be detected at future colliders.
\end{abstract}

\section{INTRODUCTION}

The existence of additional fermions, and their possible mixing with the known quarks and leptons of the Standard Model (SM), has been widely studied in the literature. These additional fermions could behave like the SM ones, as in the case of a fourth family (some times also called sequential fermions), or have exotic properties (like vectorlike or mirror fermions). In particular, mirror fermions are very interesting objects from a phenomenological point of view, and their low-energy effects have been studied too [1], including a discussion of limits on their masses [2. More recently, models involving mirror fermions generations, i.e., fermions with "mirror" isospin charges, have been discussed within a left-right (LR) symmetric context [3]. These models offer a possible solution to the strong CP problem, and have also been discussed in the context of strongly-interacting electroweak symmetry breaking scheemes [4, 䜣. Applications to the search for new astrophysical phenomena have been pursued too [6, 7].

In addition to direct production, these new fermions can manifest themselves through their mixing with the standard fermions, which can affect several aspects of flavor physics. For instance, new sources of flavor changing neutral process are usually presented in these extensions of the SM, which could lead to observable signatures and to seriously constrain such models. Most applications have focused on the light fermions, and only recently the possible implications for the top quark have been studied [8, 9]. Given the fact that coming stages of Tevatron (Run-II) will produce about $10^{4}$ top pairs, it is interesting to study rare decays of the top as possible tests of the SM [10, 11]. Furthermore, since Higgs bosons could also be produced at significant rates at Tevatron, it is also important to search for all the relevant Higgs signatures that could be detected at Run-2; future colliders (LHC, VLHC, linear colliders) will have the chance to extend further the search range. Possible rare flavor violating Higgs decay modes have been overlooked thus far, and only recently their phenomenological relevance has been discussed [12].

In this paper we analyze lepton-flavor-violating (LFV) signals in the context of LR models with mirror fermions; we focus on the mixing phenomena that arises from the interactions between the standard and exotic fermions, and their implications for the scalar Higgs sector. After discussing general conditions for the appearance of mixing in the extended fermion sector (Sec. II), we present in Sec. III the LR model with mirror fermions, which is one of the simplest LR model that solves the strong CP problem. The model contains the gauge group $\mathrm{SU}(2)_{\mathrm{L}} \times \mathrm{SU}(2)_{\mathrm{R}} \times \mathrm{U}(1)$. We discuss both its fundamental aspects and how the mixing between standard and mirror fermions can be described within the formalism presented in Sec. II. In this model we can identify two sources that can mediate flavor-changing transitions, namely: the exchange of family-violating neutral gauge bosons, and the exchange of Higgs bosons with flavor-violating couplings. We shall prove that these sources are described by independent parameters, and in order to search for the optimal rates for the signals of our interest, we shall assume that the bounds are saturated by the scalar exchange, which could simple mean that the mass of the additional gauge boson are much heavier than the

\footnotetext{
*ucotti@ifm.umich.mx

${ }^{\dagger}$ ldiaz@sirio.ifuap.buap.mx

${ }^{\ddagger}$ rgaitan@servidor.unam.mx
} 
SM-like Higgs boson of the model.

In Sec. IV we derive bounds on the parameters of the model associated with LFV transitions, for which we shall include the Higgs contributions to the transitions with strongest bounds known, namely $\mu \rightarrow e \gamma, \tau \rightarrow \mu \gamma, \tau \rightarrow e \gamma$, $\mu \rightarrow e e e, \tau \rightarrow e e e, \mu e e, \mu \mu e, \mu \mu \mu$, and electron-muon conversion in nuclei. In Sec. V we use the allowed range on the couplings $H l_{i} l_{j}$, to evaluate the B.R. for the LFV decay modes of the Higgs themselves, namely $H \rightarrow l_{i} l_{j}$, as well as the prospects to detect this signal at the future colliders (Tevatron, LHC and VLHC). Finally, Sec. VI contains our conclusions.

\section{FERMION MIXING AND FLAVOR VIOLATION}

We shall present first a generic description of fermion mixing, when two types of fermions are included: ordinary $(o)$ and exotic $(e)$. The ordinary fermions include the SM ones, whereas the exotic ones include any new fermion with sequential, mirror or singlet properties, beyond the SM. We shall assume that the SM Higgs gives masses to the SM fermions, and also that there could be an unspecified Higgs sector responsible for the exotic fermion masses, although in some cases gauge-invariant mass terms could be allowed.

To consider the mixing of fermions, we shall follow Ref. [13, grouping all fermions of a given electric charge $q$ and a helicity $a=L, R$ into $n_{a}+m_{a}$ vector column of $n_{a}$ ordinary $(o)$ and $m_{a}$ exotic $(e)$ gauge eigenstates, i.e. $\psi_{a}^{o}=\left(\psi_{o}^{o}, \psi_{e}^{o}\right)_{a}^{\top}$. The VEV of the neutral SM scalar produces the SM fermion mass terms, which together with the exotic mass and mixing matrices, will be grouped as (working in the gauge eigenstate basis):

$$
\mathcal{L}_{\text {mass }}=\overline{\psi_{L}^{o}} \mathrm{M} \psi_{R}^{o}+\text { h.c.. }
$$

The mass matrix $\mathrm{M}$ takes the form

$$
\mathrm{M}=\left(\begin{array}{ll}
\mathrm{K} & \mu^{\prime} \\
\mu & \mathrm{K}^{\prime}
\end{array}\right)
$$

where $\mathrm{K}$ denotes the SM fermion mass matrix and $\mathrm{K}^{\prime}$ corresponds to the fermion mass matrices associated with the exotic sector, while $\mu, \mu^{\prime}$ correspond to the possible mixing terms between ordinary and exotic fermions.

The form of the mass matrix will of course depend on the type of exotic fermion to be considered. Some interesting cases where one can discuss which terms arise from Spontaneous Symmetry Breaking (SSB) and which ones could be vectorlike mass terms, include the following:

- For a sequential fourth family, $\psi_{e L} \sim \mathbf{2}$ and $\psi_{e R} \sim \mathbf{1}$ under $S U(2)_{L}$, then all of $\mu, \mu^{\prime}, \mathrm{K}, \mathrm{K}^{\prime}$ arises from SSB, and become part of the full $4 \times 4$ mass matrix, which describes fermion masses and mixing.

- For a trivial vector-like representation, where: $\psi_{e L} \sim \mathbf{1}$ and $\psi_{e R} \sim \mathbf{1}$ under $S U(2)_{L}$, the terms $\mu, \mu^{\prime}$ will require $\mathrm{SSB}$, but $\mathrm{K}^{\prime}$ would be a gauge-invariant mass term.

- For a vector-like representation under the SM gauge group, i.e. with $\psi_{e L} \sim \mathbf{n}$ and $\psi_{e R} \sim \overline{\mathbf{n}}$ under $S U(2)_{L}$, then when $(\mathbf{n} \neq \mathbf{2}), \mu=\mu^{\prime}=0$ and $\mathrm{K}^{\prime}$ will be a gauge-invariant mass term.

- For a mirror family, i.e. $\psi_{e L} \sim \mathbf{1}$ and $\psi_{e R} \sim \mathbf{2}$, under $S U(2)_{L}$, then the mass terms $\mu, \mu^{\prime}$ will be gauge-invariant terms, while $\mathrm{K}^{\prime}$ will require SSB.

- For the case when $\psi_{e L} \simeq \mathbf{1}$ and $\psi_{e R} \simeq \mathbf{1}$ under $S U(2)_{L}$, but now these fermions transform non-trivially under an additional gauge group, then $\mathrm{K}^{\prime}$ will require SSB, and $\mu$ or $\mu^{\prime}$ would be the allowed gauge-invariant mass terms. This is in fact the case that will be discussed in the next section, where the exotic fermions have mirror properties with respect to a new $S U(2)$ gauge group.

The relation between the gauge eigenstates and the corresponding light $(l)$ and heavy $(h)$ mass eigenstates $\psi_{a}=$ $\left(\psi_{l}, \psi_{h}\right)_{a}^{\top}$ is given by a the transformation

$$
\psi_{a}^{o}=\mathrm{U}_{a} \psi_{a}
$$

where

$$
\mathrm{U}_{a}=\left(\begin{array}{cc}
\mathrm{A}_{a} & \mathrm{E}_{a} \\
\mathrm{~F}_{a} & \mathrm{G}_{a}
\end{array}\right) .
$$


From the unitarity of $U$

$$
\left(\mathrm{U}_{\mathrm{a}} \mathrm{U}_{\mathrm{a}}^{\dagger}\right)=1
$$

it follows that the submatrix $A_{a}$ is not unitary. The term $\left(F^{\dagger} F\right)_{a}$, which is second order in the small mirror-standard fermion mixing, will induce FC transitions in the light-light sector [29].

The diagonal mass matrix $\mathrm{M}_{\mathrm{D}}$ can be obtained through a biunitary rotation acting on the $\mathrm{L}$ and $\mathrm{R}$ sectors, namely:

$$
\mathrm{M}_{D}=\mathrm{U}_{L}^{\dagger} \mathrm{MU}_{R}=\left(\begin{array}{cc}
\mathrm{m}_{l} & 0 \\
0 & \mathrm{~m}_{h}
\end{array}\right)
$$

where $\mathrm{m}_{l}, \mathrm{~m}_{h}$ denote the light and heavy diagonal mass matrices, respectively. We will also follow Ref. [14, 15] to describe the mixing effects, though our work will concentrating mainly on the scalar flavor-changing transitions. After substituting the expression for mass-eigenstates eq. (6), we can write the matrices $\mathrm{K}, \mathrm{K}^{\prime}, \mu$ and $\mu^{\prime}$ in terms of the mass-eigenvalues and the blocks of matrix $\mathrm{U}$, as follows

$$
\begin{gathered}
\mathrm{K}=\left(\mathrm{A}_{L} \mathrm{~m}_{l} \mathrm{~A}_{R}^{\dagger}+\mathrm{E}_{L} \mathrm{~m}_{h} \mathrm{E}_{R}^{\dagger}\right), \\
\mathrm{K}^{\prime}=\left(\mathrm{F}_{L} \mathrm{~m}_{l} \mathrm{~F}_{R}^{\dagger}+\mathrm{G}_{L} \mathrm{~m}_{h} \mathrm{G}_{R}^{\dagger}\right), \\
\mu=\mathrm{F}_{L} \mathrm{~m}_{l} A_{R}^{\dagger}+\mathrm{G}_{L} \mathrm{~m}_{h} E_{R}^{\dagger}, \\
\mu^{\prime}=\mathrm{A}_{\mathrm{L}} \mathrm{m}_{\mathrm{l}} \mathrm{F}_{\mathrm{R}}^{\dagger}+\mathrm{E}_{\mathrm{L}} \mathrm{m}_{\mathrm{h}}^{\prime} \mathrm{G}_{\mathrm{R}}^{\dagger} .
\end{gathered}
$$

When one describes the scalar-fermion couplings within some specific Higgs sector, it turns out that those couplings will not be diagonal in general, thus new FCNC phenomena associated will be predicted in such model. It is relevant to mention here, that most analysis of FCNC/LFV process in extended gauge models, have concentrated on the flavor changing vertices of the additional neutral gauge bosons, but the Higgs sector has been mostly overlooked. As it will be shown in the next sections, FCNC phenomena associated with the scalar sector can produce new discovery signals, consistent with the current bounds on FCNC/LFV transitions. The resulting rates could be detected at coming stages of colliders (RUN-2 of Tevatron) or in the future ones (LHC and VLHC). The specific Higgs sector that we will work with, corresponds to the minimal LR model with mirror fermions. This model and the resulting phenomenological constraints, will be discussed in the coming sections.

\section{FERMION MIXING IN A LEFT-RIGHT MODEL WITH MIRROR FERMIONS}

A first speculation on the existence of mirror fermions appeared in the classical paper on parity violation that led to the V-A interactions models [16]. On the other hand, the left-right symmetric models renders the baryon-lepton number symmetry $\mathrm{U}(1)_{\mathrm{B}-\mathrm{L}}$ more natural by gauging it, and it has also been proposed as a solution to the strong $\mathrm{CP}$ problem when accompanied by the introduction of mirror fermions 17, 18. Such an extended gauge group with additional fermions, apart from fitting nicely into unification schemata, restores the left-right symmetry missing in the Standard Model (SM) in a manner that goes beyond the simplest left-right symmetry models.

In the left-right model with mirror fermions (LRMM) [3], the right-handed (left-handed) components of mirror fermions transform as doublets (singlets) under the new $\mathrm{SU}(2)_{\mathrm{R}}$. The $\mathrm{SM}$ fermions are singlets under the $\mathrm{SU}(2)_{\mathrm{R}}$, whereas the right-handed mirror are also singlets under $\mathrm{SU}(2)_{\mathrm{L}}$. Mirror and SM fermions will share hypercharge and color interactions. Thus, the first family of leptons and quarks will be written as follows:

$$
\begin{array}{rlrl}
l_{e L}^{o} & =\left(\begin{array}{l}
\nu_{\mathrm{e}}^{\mathrm{o}} \\
\mathrm{e}^{\mathrm{o}}
\end{array}\right)_{L}, \quad e_{R}^{o}, & l^{\prime o}=\left(\begin{array}{c}
\nu^{\prime o} \\
e^{\prime o}
\end{array}\right)_{R}, \quad e_{L}^{\prime o} \\
q_{L}^{o}=\left(\begin{array}{c}
u^{o} \\
d^{o}
\end{array}\right)_{L}, \quad u_{R}^{o}, d_{R}^{o}, & q_{R}^{\prime o}=\left(\begin{array}{c}
u^{\prime o} \\
d^{\prime o}
\end{array}\right)_{R}, u_{L}^{\prime o}, d_{L}^{\prime o}
\end{array}
$$


The superscript $(o)$ denote weak eigenstates, and the primes will be associated with the mirror particles. Because the model does not contain left-handed mirror neutrinos, they have to be massless. Extensions of the model to include neutrino masses has been considered too [7], but it is beyond the scope of our present work.

subsectionSymmetry Breaking The symmetry breaking is realized by including two Higgs doublets, the SM one $(\phi)$ and its mirror partner $\left(\phi^{\prime}\right)$. The potential of the model can be written in a such a way that the vacuum expectation values (VEV's) of the Higgs field are

$$
\langle\phi\rangle=\frac{1}{\sqrt{2}}\left(\begin{array}{l}
0 \\
v
\end{array}\right), \quad\left\langle\phi^{\prime}\right\rangle=\frac{1}{\sqrt{2}}\left(\begin{array}{l}
0 \\
v^{\prime}
\end{array}\right) .
$$

The most general potential that develops this pattern of VEV's is:

$$
V=-\left(\mu_{1}^{2} \phi^{\dagger} \phi+\mu_{2}^{2} \phi^{\prime \dagger} \phi^{\prime}\right)+\frac{\lambda_{1}}{2}\left(\left(\phi^{\dagger} \phi\right)^{1}+\left({\phi^{\prime}}^{\dagger} \phi^{\prime}\right)^{2}\right)+\lambda_{2}\left(\phi^{\dagger} \phi\right)\left(\phi^{\dagger} \phi\right) .
$$

The terms with $\mu_{1}, \mu_{2}$ are included so that the parity symmetry $(\mathrm{P})$ is broken softly, i.e. only through the dimensiontwo mass terms of the Higgs potential.

The neutral Higgs boson squared mass matrix that follows from this potential is:

$$
\mathrm{M}_{\mathrm{H}^{0}}^{2}=\left(\begin{array}{cc}
2 \lambda_{1} v^{2} & 2 \lambda_{2} v v^{\prime} \\
2 \lambda_{2} v v^{\prime} & 2 \lambda_{1} v^{\prime 2}
\end{array}\right)
$$

Diagonalization of the higgs-boson squared mass matrix is straightforward using a real basis. Out of the eight scalar degrees of freedom associated with two complex doublets, six become the Goldstone bosons required to give mass to $W^{ \pm}, W^{\prime \pm}, Z$ and $Z^{\prime}$. Thus only two neutral Higgs bosons remain; the neutral physical states are:

$$
\begin{array}{r}
H=\sqrt{2}\left(\left(\Re e \phi^{o}-v\right) \cos \alpha+\left(\Re e \phi^{\prime o}-v^{\prime}\right) \sin \alpha\right), \\
H^{\prime}=\sqrt{2}\left(-\left(\Re e \phi^{o}-v\right) \sin \alpha+\left(\Re e \phi^{\prime o}-v^{\prime}\right) \cos \alpha\right),
\end{array}
$$

where $\alpha$ denotes the neutral Higgs mixing angle.

The mass matrix for the gauge bosons is obtained from the scalar Lagrangian

$$
\mathcal{L}_{\text {sca }}=\left(\mathrm{D}^{\mu} \phi\right)^{\dagger}\left(\mathrm{D}_{\mu} \phi\right)+\left(\mathrm{D}^{\prime \mu} \phi^{\prime}\right)^{\dagger}\left(\mathrm{D}_{\mu}^{\prime} \phi^{\prime}\right)
$$

where $D_{\mu}$ denotes the covariant derivative associated with the SM, and $D_{\mu}^{\prime}$ is the one associated with the mirror sector.

After substituting the vev's (12) in the Lagrangian (17) we obtain the expressions for the mass matrices. The mass matrix for the charged gauge bosons is already diagonal, with mass eigenvalues: $M_{W}=\frac{1}{2} v g_{2}$ and $M_{W}=\frac{1}{2} v^{\prime} g_{2}^{\prime}$, where $g_{2}$ and $g_{2}^{\prime}$ are the coupling constant associated with the $\mathrm{SU}(2)_{\mathrm{L}}$ and $\mathrm{SU}(2)_{\mathrm{R}}$ gauge group, respectively. The mass matrix for the neutral gauge bosons is not diagonal, and is given by

$$
\frac{1}{4}\left(\begin{array}{ccc}
g_{2}^{2} & 0 & -g_{2} g_{1} v^{2} \\
0 & g_{2}^{\prime 2} v^{\prime 2} & -g_{2}^{\prime} g_{1} v^{\prime 2} \\
-g_{2} g_{1} v^{2} & g_{2}^{\prime} g_{1} v^{\prime 2} & g_{1}^{2}\left(v^{2}+v^{\prime 2}\right.
\end{array}\right)
$$

However this matrix can be diagonalized by an orthogonal transformation R, which relates the weak and mass eigenstates, namely [3]

$$
\mathrm{R}=\left(\begin{array}{ccc}
c_{\theta_{w}} c_{\Theta} & s_{\theta_{w}} \\
-\frac{1}{c_{\theta_{w}}}\left(s_{\Theta} r_{\theta_{w}}+\frac{g_{2}}{g_{2}^{\prime}} c_{\Theta} s_{\theta_{w}}^{2}\right) & \frac{1}{c_{\theta_{w}}}\left(c_{\Theta} r_{\theta_{w}}-\frac{g_{2}}{g_{2}^{\prime}} s_{\Theta} s_{\theta_{w}}^{2}\right) & \frac{g_{2}}{g_{2}^{\prime}} s_{\theta_{w}} \\
t_{\theta_{w}}\left(\frac{g_{2}}{g_{2}^{\prime}} s_{\Theta}-r_{\theta_{w}} c_{\Theta}\right) & -t_{\theta_{w}}\left(\frac{g_{2}}{g_{2}^{\prime}} c_{\Theta}+r_{\theta_{w}} s_{\Theta}\right) & r_{\theta_{w}}
\end{array}\right)
$$

with $\theta_{w}$ and $\Theta$ denoting the rotations angles of the neutral gauge bosons. In eq. (19), $r_{\theta_{w}} \equiv \sqrt{c_{\theta_{w}}^{2}-\frac{g_{2}^{2}}{{g^{\prime}}_{2}^{2}} s_{\theta_{w}}^{2}}$. One has one massless state (the photon) and two massive states, with eigenvalues given by

$$
M_{Z, Z^{\prime}}=\frac{1}{2}\left(v^{2}\left(g_{2}^{2}+g_{1}^{2}\right)+v^{\prime 2}\left(g_{2}^{\prime 2}+g_{1}^{2}\right)\right) \mp \frac{1}{2} \sqrt{\left(v^{2}\left(g_{2}^{2}+g_{1}^{2}\right)+v^{\prime 2}\left(g^{\prime 2}+g_{1}^{2}\right)\right)^{2}-4 v^{2} v^{\prime 2}\left(g_{2}^{2} g_{1}^{2}+g_{2}^{2} g_{2}^{\prime 2}+g_{2}^{\prime 2} g_{1}^{2}\right)}
$$


where $g_{1}$ is the coupling constant of the $U(1)$ gauge group.

In order to find the couplings of the Higgs fields with the neutral gauge boson, we expand the expressions for $D_{\mu}$ and $D_{\mu}^{\prime}$, substituting the physical states into eq. (17). We get the following expression for the $Z Z H / H^{\prime}$ interactions:

$$
\begin{array}{r}
\mathcal{L}_{Z Z H}=\sqrt{2}\left(g_{2} M_{W} X\left(\Theta, \theta_{w}\right) \cos \alpha+g_{2}^{\prime} M_{W^{\prime}} Y\left(\Theta, \theta_{w}\right) \sin \alpha\right) H Z_{\mu} Z^{\mu} \\
+\sqrt{2}\left(-g_{2} M_{W} X\left(\Theta, \theta_{w}\right) \sin \alpha+g_{2}^{\prime} M_{W^{\prime}} Y\left(\Theta, \theta_{w}\right) \cos \alpha\right) H^{\prime} Z_{\mu} Z^{\mu}
\end{array}
$$

where

$$
\begin{gathered}
X\left(\Theta, \theta_{w}\right)=\left(c_{\theta_{w}} c_{\Theta}-\frac{g_{1}}{g_{2}} t_{\theta_{w}}\left(\frac{g_{2}}{g_{2}^{\prime}} s_{\Theta}-r_{\theta_{w}} c_{\Theta}\right)\right)^{2} \\
Y\left(\Theta, \theta_{w}\right)=\left(-\frac{1}{c_{\theta_{w}}}\left(s_{\Theta} r_{\theta_{w}}+\frac{g_{2}}{g_{2}^{\prime}} c_{\Theta} s_{\theta_{w}}^{2}\right)-\frac{g_{1}}{g_{2}} t_{\theta_{w}}\left(\frac{g_{2}}{g_{2}^{\prime}} s_{\Theta}-r_{\theta_{w}} c_{\Theta}\right)\right)^{2}
\end{gathered}
$$

On the other hand, the expressions for the Higgs-charged gauge boson interactions $\left(W W H / H^{\prime}\right)$ are given by:

$$
\mathcal{L}_{W W H}=\sqrt{2} g_{2} M_{W} g^{\mu \nu}\left(H \cos \alpha-H^{\prime} \sin \alpha\right) W_{\mu}^{-} W_{\nu}^{+}
$$

\section{A. Yukawa Lagrangian and fermion mixing}

The renormalizable and gauge invariant interactions of the scalar doublets $\phi$ and $\phi^{\prime}$ with the leptons are described by the Yukawa Lagrangian, which takes the form

$$
\mathcal{L}_{Y}^{l}=\lambda_{i j} \bar{l}_{i L}^{o} \phi e_{j R}^{o}+\lambda_{i j}^{\prime}{\overline{l^{\prime}}}_{i R}^{o} \phi^{\prime}{e^{\prime o}}_{j L}^{o}+\mu_{i j}{\overline{e^{\prime}}}_{i L}^{o} e_{j R}^{o}+h . c .
$$

where $i, j=1,2,3$ and $\lambda_{i j}, \hat{\lambda}_{i j}$, and $\mu_{i j}$ are (unknown) matrices.

For the quarks fields, the corresponding Yukawa terms are written as

$$
\mathcal{L}_{Y}^{q}=\lambda_{i j}^{d} \bar{Q}_{i L}^{o} \phi d_{j R}^{o}+\lambda_{i j}^{u} \bar{Q}_{i L}^{o} \tilde{\phi} u_{j R}^{o}+\lambda^{\prime}{ }_{i j}{\overline{Q^{\prime}}}_{i R}^{o} \phi^{\prime} d^{\prime o}{ }_{j L}+\lambda^{\prime u}{\overline{Q^{\prime}}}_{i R}^{o}{\tilde{\phi^{\prime}}}_{u^{\prime}}^{\prime o}+\mu_{i j}^{d}{\overline{d^{\prime}}}_{i L}^{o} d_{j R}^{o}+\mu_{i j}^{u}{\overline{u^{\prime}}}_{i L}^{o} u_{j R}^{o}+h . c .
$$

the conjugate fields $\tilde{\phi}\left(\tilde{\phi}^{\prime}\right)$ are obtained as $\tilde{\phi}=i \tau_{2} \phi^{*}$.

The VEV's of the neutral scalars produce the fermion mass terms, which in the gauge eigenstate basis read

$$
\mathcal{L}_{\text {mass }}=\overline{\psi_{L}^{o}} \mathrm{M} \psi_{R}^{o}+\text { h.c. }
$$

For the lepton sector, the non-diagonal mass matrix $\mathrm{M}$, takes the form

$$
\mathrm{M}=\left(\begin{array}{cc}
\mathrm{K} & 0 \\
\mu & \mathrm{K}^{\prime}
\end{array}\right)
$$

where $\mathrm{K}=\frac{1}{2} \lambda \mathrm{v}$ and $\mathrm{K}^{\prime}=\frac{1}{2} \lambda^{\prime} \mathrm{v}^{\prime}$ correspond to the $3 \times 3$ matrices generated from the symmetry breaking VEV's; $\mu$ corresponds to the gauge invariant $3 \times 3$ mixing terms between ordinary and mirror fermions singlets. Thus, we have $\mu^{\prime}=0$ in Eq. (2), and then:

$$
\mathrm{M}_{D}=\mathrm{U}_{L}^{\dagger} \mathrm{MU}_{R}
$$

With the help of the relations (7-10), and working within the Higgs mass-eigenstate basis, the tree-level interactions of the neutral Higgs bosons $\mathrm{H}$ and $\mathrm{H}$ ' with the light fermions are given by

$$
\mathcal{L}_{Y}^{l}=\frac{g_{2}}{2 \sqrt{2}} \bar{f}_{L} \mathrm{~A}_{L}^{\dagger} \mathrm{A}_{L} \frac{m_{l}}{M_{W}} f_{R}\left(H \cos \alpha-H^{\prime} \sin \alpha\right)+\frac{g_{2}^{\prime}}{\sqrt{2}} \bar{f}_{L} \frac{m_{l}}{M_{W^{\prime}}} \mathrm{F}_{R}^{\dagger} \mathrm{F}_{R} f_{R}\left(H \sin \alpha+H^{\prime} \cos \alpha\right)+\text { h.c.. }
$$

One can see that the couplings are not diagonal in general, thus new phenomena associated with FCNC will be present in this model. The resulting phenomenological constraints and predictions will be discussed in the coming sections.

Finally, once we have obtained the quark and lepton mass eigenstates, their gauge interaction can be obtained from the Lagrangian

$$
\mathcal{L}^{\text {int }}=\bar{\psi} i \gamma^{\mu} D^{\mu} \psi+\overline{\psi^{\prime}} i \gamma^{\mu} D_{\mu}^{\prime} \psi^{\prime}
$$


where $\psi, \psi^{\prime}$ denote the standard and mirror fermions, respectively.

The neutral current term for the multiplet $\psi$ of a given electric charge, including the contribution of the neutral gauge boson mixing, can be written as follows

$$
-\mathcal{L}^{\mathrm{nc}}=\sum_{a=L, R} \bar{\psi}_{a}^{o} \gamma^{\mu}\left(g_{2} \boldsymbol{\top}_{3 a}, g_{2}^{\prime} \mathrm{T}_{3 a}^{\prime}, g_{1} \frac{\mathrm{Y}_{a}}{2}\right) \psi_{a}^{o}\left(\begin{array}{l}
W^{3} \\
W^{\prime 3} \\
B
\end{array}\right)_{\mu}
$$

Using Eqs. (3,4), one arrives to the following expression in terms of the mass eigenstates:

$$
-\mathcal{L}^{\mathrm{nc}}=\sum_{a=L, R} \bar{\psi}_{a} \gamma^{\mu} \mathrm{U}_{a}^{\dagger}\left(g_{2} \mathrm{~T}_{3 a}, g_{2}^{\prime} \mathrm{T}^{\prime}{ }_{3 a}, g_{1} \frac{\mathrm{Y}_{a}}{2}\right) \mathrm{U}_{a} \psi_{a}\left(\begin{array}{c}
Z \\
Z^{\prime} \\
A
\end{array}\right)_{\mu}
$$

where $\mathrm{T}_{3 a}, \mathrm{~T}^{\prime}{ }_{3 a}$, and $\mathrm{Y}$ are the generators of the $\mathrm{SU}(2)_{\mathrm{L}}, \mathrm{SU}(2)_{\mathrm{R}}$, and $\mathrm{U}(1)$, respectively.

As we mentioned before, in the coming analysis we shall concentrate on the scalar mediated flavour-violating transitions, assuming that they saturate current bounds on FCNC/LFV transitions, which will also simplify the analysis.

\section{CONSTRAINTS FROM LFV TRANSITIONS MEDIATED BY THE HIGGS BOSON}

In the following, we shall discuss the constraints that low-energy data imposes on the parameters of the LFV higgs lagrangian. After neglecting the lighter lepton masses in each vertex, we arrive to the following expressions for the LFV Higgs interactions:

$$
\left(H l_{i} l_{j}\right): \frac{g_{2} m_{j}}{2 m_{W}} \eta_{i j} P_{R}
$$

with $m_{j}>m_{i}$ and $P_{R}=\left(1+\gamma_{5}\right) / 2$. For the diagonal interactions we obtain,

$$
\left(H l_{i} l_{i}\right): \frac{g_{2} m_{i}}{2 m_{W}} \eta_{i i}
$$

In eqs. (34) and (35), $\eta_{i j}=\left(A_{L}^{\dagger} A_{L}\right)_{i j} \cos \alpha$. In fact, we shall see that the resulting bounds can be expressed in terms of the parameters $\eta_{i j} / m_{H}^{2}$.

\section{A. The decays $l_{i} \rightarrow l_{j} l_{k} l_{k}$}

The flavor-changing interactions of the scalars $H$ and $H^{\prime}$ will mediate the lepton number violation decays $l_{i} \rightarrow l_{j} l_{k} l_{k}$. By assuming $m_{H^{\prime}}>>m_{H}$, one can neglect the contribution of the mirror Higgs. Then, using the interactions contained in eqs.(35-36) we can derive the expressions for the decay width of these processes. The result is given by:

$$
\begin{array}{r}
\Gamma=\frac{m_{i}^{5}}{3072 \pi^{3} M_{H}^{4}}\left(\left(\left|g_{V}^{i k}\right|^{2}+\left|g_{A}^{i k}\right|^{2}\right)\left(\left|g_{V}^{j k}\right|^{2}+\left|g_{A}^{j k}\right|^{2}\right)+\left(\left|g_{V}^{i j}\right|^{2}+\left|g_{A}^{i j}\right|^{2}\right)\left(\left|g_{V}^{k k}\right|^{2}\right)+\right. \\
\left.\frac{1}{2}\left(g_{V}^{i k} g_{V}^{j k} g_{V}^{i j} g_{V}^{k k}+g_{V}^{i k} g_{V}^{k k} g_{A}^{j k} g_{A}^{i j}+g_{V}^{j k} g_{V}^{k k} g_{A}^{i k} g_{A}^{i j}+g_{V}^{i j} g_{V}^{k k} g_{A}^{i k} g_{A}^{j k}\right)\right) .
\end{array}
$$

This amplitude includes two possible Feynman graphs, one with two LFV vertices and one with a flavor conserving vertex. The explicit expressions for the scalar $g_{V}^{i j}$ and pseudoscalar $g_{A}^{i j}$ couplings associated with the lepton transitions $i j$ (with $i \neq j$ ) mediated by the scalar $H$, are given by:

$$
g_{V}^{i j}=g_{A}^{i j}=\frac{g_{2}}{4 M_{W}} \eta_{i j} m_{j}
$$

whereas

$$
g_{V}^{k k}=\frac{g_{2}}{2 M_{W}} \eta_{k k} m_{j}, \quad g_{A}^{k k}=0 .
$$


In deriving Eq. (36), we have neglected the lepton masses of the final states. On the other hand, we have neglected the $Z$-fermions interactions because they are diagonal in this approximation.

Now, we examine the three-body decays $\mu \rightarrow$ eee, $\tau \rightarrow$ eee, $\tau \rightarrow \mu \mu \mu, \tau \rightarrow \mu \mu \mathrm{e}, \tau \rightarrow$ ee $\mu, \tau \rightarrow$ e $\mu$ e, and $\tau \rightarrow$ e $\mu \mu$, which can be induced by the $L F V$ couplings. Using the expressions for the partial withs and comparing with the experimental limits, we derive bounds on the flavor changing couplings $\eta_{\mathrm{ij}}$ as function of $M_{H}$. The results are:

1. $\mu \rightarrow$ eee

$$
\eta_{e e}^{2} \eta_{\mu e}^{2}<5.5 \times 10^{-3} M_{H}^{4} G e V^{-4}
$$

2. $\tau \rightarrow$ eee

$$
\eta_{e e}^{2} \eta_{\tau e}^{2}<3.2 \times 10^{2} M_{H}^{4} G e V^{-4}
$$

3. $\tau \rightarrow \mu \mu \mu$

$$
\eta_{\mu \mu}^{2} \eta_{\tau \mu}^{2}<4.8 \times 10^{-3} M_{H}^{4} G e V^{-4}
$$

4. $\tau \rightarrow \mu \mu e$

$$
\eta_{\tau \mu}^{2} \eta_{\mu e}^{2}<7.6 \times 10^{-3} M_{H}^{4} G e V^{-4}
$$

5. $\tau \rightarrow e e \mu$

$$
\eta_{\tau e}^{2} \eta_{\mu e}^{2}<7.6 \times 10^{-3} M_{H}^{4} G e V^{-4}
$$

6. $\tau \rightarrow e \mu e$

$$
\eta_{e e}^{2} \eta_{\tau \mu}^{2}+2 \times 10^{4} \eta_{\tau e}^{2} \eta_{\mu e}^{2}+2 \times 10^{2} \eta_{e e} \eta_{\tau e} \eta_{\mu e} \eta_{\tau \mu}<2 \times 10^{2} M_{H}^{4} G e V^{-4}
$$

7. $\tau \rightarrow e \mu \mu$

$$
\eta_{\tau e}^{2} \eta_{\mu \mu}^{2}+\frac{1}{2} \eta_{\tau \mu}^{2} \eta_{\mu e}^{2}+\frac{1}{2} \eta_{\tau e} \eta_{\mu \mu} \eta_{\mu e} \eta_{\tau \mu}<4.6 \times 10^{-3} M_{H}^{4} G e V^{-4}
$$

For instance, taking $M_{H}=130 \mathrm{GeV}$ gives the following bounds: $\eta_{e e}^{2} \eta_{\mu e}^{2}<1.6 \times 10^{6}, \eta_{\mu \mu}^{2} \eta_{\mu \tau}^{2}<1.4 \times 10^{6}, \eta_{\tau \mu}^{2} \eta_{\mu e}^{2}<$ $2.2 \times 10^{6}$, and $\eta_{\tau e}^{2} \eta_{\mu e}^{2}<2.2 \times 10^{6}$. These results show that the LFV Higgs couplings are essentially unconstrained by low-energy phenomenology. However, since they appear as products of fermion and Higgs mixing angles, it is natural to expect them to be naturally of order 1; moreover, for the application to LFV Higgs decay, we shall take them conservatively to be of order 0.1 .

\section{B. Radiative decays}

Here we analyze the lepton flavor violation processes $\mu \rightarrow e \gamma, \tau \rightarrow e \gamma$ and $\tau \rightarrow \mu \gamma$, arising in the model as a consequence of the existence of the gauge invariant mixing terms $\mu_{\mathrm{ij}} \overline{\mathrm{e}}_{\mathrm{iL}}^{o} \mathrm{e}_{\mathrm{jR}}^{o}+$ h.c of ordinary leptons with mirror counterparts. We discuss here the main details for the process $\mu \rightarrow e \gamma$, with the other processes being computed in an analogous way.

The lowest order contribution to the $\mu \rightarrow e \gamma$ decay mediated by the neutral scalar fields comes from the Feynman diagrams where the photon is radiated from an internal lepton line. As it is known the corresponding amplitude is proportional to the operator $\overline{\mathrm{u}\left(\mathrm{p}_{2}\right)} \sigma^{\mu \nu} q_{\nu} \epsilon_{\mu} \mathrm{u}\left(\mathrm{p}_{1}\right)$, where $q=p_{1}-p_{2}$ and $\epsilon_{\mu}$ is the polarization of the photon; an helicity flip is therefore involved. The amplitudes obtained by computing the one loop diagrams are

$$
i \mathrm{~m}_{\mathrm{A}}=C_{A} \bar{e} i \frac{\sigma^{\mu \nu} q_{\nu} \epsilon_{\mu}}{m_{\mu}+m_{e}} \frac{1-\gamma_{5}}{2} \mu
$$

and

$$
i \mathrm{~m}_{\mathrm{B}}=C_{B} \bar{e} i \frac{\sigma^{\mu \nu} q_{\nu} \epsilon_{\mu}}{m_{\mu}+m_{e}} \frac{1+\gamma_{5}}{2} \mu
$$


corresponding to the contributions to $\mu_{\mathrm{L}} \rightarrow e_{\mathrm{R}} \gamma$ and $\mu_{\mathrm{R}} \rightarrow e_{L} \gamma$ respectively.

From the unitary of $\mathrm{U}$ it follows that

$$
A^{\dagger} A=1-F^{\dagger} F
$$

Hence, the matrix A describing the mixing with the ordinary charged leptons is non-unitary by small terms quadratic in the ordinary-exotic charged lepton mixing present in F. In our calculation we keep only the lowest order in the non-diagonal terms. So, the leading contribution to the coefficients $C_{A}$ and $C_{B}$, in the limit $\alpha \ll 1, M_{W} \ll M_{\hat{W}}$, and $M_{H} \ll M_{H^{\prime}}$ is given by

$$
C_{A}=\frac{e}{16 \pi^{2}} \frac{g^{2}}{4 M_{H}^{2} M_{W}^{2}}\left(m_{\mu}+m_{e}\right) m_{\mu}^{2} m_{e}\left[\ln \frac{M_{H}^{2}}{m_{\mu}^{2}}-\frac{4}{3}\right]\left(\mathrm{A}_{L}^{\dagger} \mathrm{A}_{L}\right)_{12}
$$

and

$$
C_{B}=\frac{e}{16 \pi^{2}} \frac{g^{2}}{4 M_{H}^{2} M_{W}^{2}}\left(m_{\mu}+m_{e}\right) m_{\tau}^{3}\left[\ln \frac{M_{H}^{2}}{m_{\mu}^{2}}-\frac{4}{3}\right]\left(\mathrm{A}_{L}^{\dagger} \mathrm{A}_{L}\right)_{12}
$$

After comparing Eq. (46) and Eq. (47) with the general expressions from Ref. [19] for a process with a real photon $f_{1} \longrightarrow f_{2}+\gamma:$

$$
\begin{aligned}
i \mathcal{M}\left(f_{1}\left(p_{1}\right) \rightarrow f_{2}\left(p_{2}\right)+\gamma(q)\right) & =\bar{u}_{2}\left(p_{2}\right) i \frac{\sigma^{\mu \nu} q_{\nu} \epsilon_{\mu}}{m_{1}+m_{2}}\left(F(0)_{21}^{V}+F(0)_{21}^{A} \gamma_{5}\right) u_{1}\left(p_{1}\right), \\
\Gamma\left(f_{1} \rightarrow f_{2}+\gamma\right) & =\frac{m_{1}}{8 \pi}\left(1-\frac{m_{2}}{m_{1}}\right)^{2}\left(1-\frac{m_{2}^{2}}{m_{1}^{2}}\right)\left[\left|F(0)_{21}^{V}\right|^{2}+\left|F(0)_{21}^{A}\right|^{2}\right]
\end{aligned}
$$

We find in our case:

$$
\begin{aligned}
& F(0)_{21}^{V}=\frac{1}{2}\left(C_{A}+C_{B}\right), \\
& F(0)_{21}^{A}=\frac{1}{2}\left(C_{B}-C_{A}\right)
\end{aligned}
$$

and in the limit $m_{e} \ll m_{\mu} \ll m_{\tau}$ we get

$$
\Gamma\left(l_{i} \rightarrow l_{j}+\gamma\right)=\frac{\alpha_{e . m .}}{512 \pi^{4}}\left(G_{F} m_{l_{i}}^{2}\right)^{2} \frac{m_{l_{i}}^{5}}{M_{H}^{4}}\left[\ln \frac{M_{H}^{2}}{m_{l_{i}}^{2}}-\frac{4}{3}\right]^{2}\left|\left(\mathrm{~A}_{L}^{\dagger} \mathrm{A}_{L}\right)_{i j}\right|^{2}
$$

The bounds on the flavor-changing couplings which are obtained by comparing these radiative decays with current limits, are, respectively, $\eta_{e \mu}^{2}<1.9 \times 10^{2}, \eta_{\tau e}^{2}<9.2 \times 10^{3}$, and $\eta_{\tau \mu}^{2}<3.9 \times 10^{3}$, where we have taken $M_{H}=130 \mathrm{GeV}$ and $\eta_{i i} \simeq 1$.

\section{Bounds from electron-muon conversion}

Neutrinoless $\mu^{-}(A, Z) \rightarrow e^{-}(A, Z)$ conversion in muonic atoms with mass number $A$ and atomic number $Z$ offer another sensitive test of $L F V$ [20, 21, 22]. The experimental bound on the branching ratio for $\mu-e$ conversion in titanium gives a constraint on possible violation of the muon and electron numbers. In this section we obtain a bound on the parameter $\eta_{\mu e}$ implied by the limit for the $L F V$ reaction, as mediated by the light Higgs of the model. Using the results of Ref. [23], we can write:

$$
\left(\left|g_{V}^{\mu e}\right|^{2}+\left|g_{A}^{\mu e}\right|^{2}\right)^{\frac{1}{2}} \leq \frac{2.4}{\sqrt{2}} \times 10^{-7}\left(\frac{0.5 G e V}{\overline{m_{N}}}\right)\left(\frac{R}{10^{-16}}\right)^{\frac{1}{2}} \frac{M_{H}^{2}}{M_{W}^{2}}
$$

where

$$
\overline{m_{N}}=\frac{1}{2}\left(m_{u}+m_{d}\right)=5 \mathrm{MeV}
$$

is the current quark mass and $R \simeq 10^{-16}$ is the present sensitivity. Taking the values of $g_{V}^{\mu e}$ and $g_{A}^{\mu e}$ of our model, we obtain:

$$
\eta_{\mu e}^{2} \leq 8.6 \times 10^{-6} M_{H}^{2} G e V^{-2}
$$

Taking $M_{H}=130 \mathrm{GeV}$, gives the result $\eta_{\mu e}^{2} \leq 1.5 \times 10^{-1}$, which is in fact the stronger bound. 


\section{DETECTION OF THE LFV HIGGS DECAYS $\left(H \rightarrow l_{i} l_{j}\right)$}

The search for the Higgs boson is one of the main goals of Tevatron RUN-2 and future colliders [24]. Although the most conservative search strategy uses the theoretical expectations coming from the minimal standard model (SM), it is certainly worthwhile to look for other signals arising from physics beyond the SM. In this regard, it has been recognized recently that the Higgs sector of several well-motivated models can predict lepton flavor violating (LFV) Higgs decays with sizable values [12] without being in conflict with low-energy phenomenology, and at rates that may be detectable at future colliders. The case of the generic two-higgs doublet model III, has been further studied for linear and hadron colliders [25, 26], with the conclusion that it is possible to detect the LFV HIggs signal, and this also holds for the effective lagrangian extension of the SM. Within the minimal SUGRA-MSSM and the SM with massive neutrinos, these decays are found to have negligible rates. Whereas in models with heavy majorana neutrinos, the LFV Higgs decays are induced at one-loop level and the Branching ratio (B.r.) can reach values of order $10^{-3}$ [27.

In this paper we also study the detectability of the resulting LFV Higgs decays that arise in the LRMM. In addition to considering the reach of Tevatron and LHC, we also estimate the bounds on the LFV Higgs couplings that could be obtained at the VLHC.

\section{A. The Branching ratios for LFV Higgs decays}

One additional implication of these LFV couplings is the possibility to observe the LFV Higgs decays $h^{0} \rightarrow l_{i}^{+} l_{j}^{-}$, whose decay width is given by:

$$
\Gamma\left(h \rightarrow l_{i} l_{j}\right)=\frac{g_{2}^{2} m_{j}^{2} m_{H}}{12 \pi m_{W}^{2}}\left|\eta_{i j}\right|^{2}
$$

For current favored range of Higgs masses $\left(115<m_{h}<200 \mathrm{GeV}\right)$, one of the dominant decay modes of the Higgs boson is into $h \rightarrow b \bar{b}$, with the corresponding coupling being proportional to $\eta_{b b}$, and this will introduce a complicated expression for the Higgs total width. In order to handle such multi-parameter dependence, we shall neglect the deviations from the SM for the diagonal Higgs couplings. Furthermore, since the low-energy bounds only constrain significantly the coupling $h e \mu$, and the bounds on the vertex $h \tau l_{i}$ are not really significant, we have evaluated the B.r. taking the parameter $\eta_{\tau l_{i}}=0.1$; the results are shown in Fig. 11, and one can notice that B.r. $(h \rightarrow \tau \mu) \simeq 0.01$ for $m_{h} \simeq 100-160 \mathrm{GeV}$.

The mode $h \rightarrow \tau e$ has a similar branching ratio, whereas B.r. $(h \rightarrow e \mu)$ can reach at most values of the order $\simeq 10^{-5}$.

\section{B. Detection of LFV Higgs decays at hadron colliders}

In order to study these LFV Higgs decays at future hadron colliders, we shall focus on the mode $h^{0} \rightarrow \tau \mu$ which, having the muon in the final state, seems easier to separate from the backgrounds. Then, to derive bounds on the LFV parameters, we shall introduce the parameter $\kappa_{\mu \tau}$, in such a way that the branching ratio for the decay $h^{0} \rightarrow \tau \mu$, is given by:

$$
\text { B.r. }\left(h^{0} \rightarrow \tau \mu\right)=2 \kappa_{\tau \mu}^{2} \text { B.r. }\left(h_{S M} \rightarrow \tau^{+} \tau^{-}\right)
$$

where the dependence on the parameters $\eta_{i j}$ has been absorbed into the couplings $\kappa_{\tau \mu}$.

Thus, given that we naturally expect this mode to have a b.r. in the range $10^{-2}-10^{-1}$, it seems quite interesting to try looking for the prospectives to detect these modes at future colliders. An estimate of their feasibility at Tevatron in ref. [12], was followed by a more detailed background study in ref. [26], where the LHC case was included too. The case of a linear colliders was studied in ref. 25]. In this paper, we shall also include the case of a Very Large Hadron Collider, with a c.m. energy of $40 \mathrm{TeV}$.

In order to study the possibility to detect the LFV higgs decays, one can use the gluon-fusion mechanism to produce a single Higgs boson; assuming that the production cross-section is of similar strength to the SM case, about $1.2 \mathrm{pb}$ for $m_{h}=100 \mathrm{GeV}$, it will allow to produce 12,000 Higgs bosons with an integrated luminosity of $10 \mathrm{fb}^{-1}$. Thus, for B.R. $(h \rightarrow \tau \mu / \tau e) \simeq 10^{-1}-10^{-2}$ Tevatron can produce 1200-120 events. Then, to determine the detectability of the signal, we need to study the main backgrounds to the $h \rightarrow \tau \mu$ signal, which are dominated by Drell-Yan tau pair and WW pair production. In Ref. [26] it was proposed to reconstruct the hadronic and electronic tau decays, assuming the following cuts: i) For the transverse muon and jet momentum: $p_{T}^{\mu}>m_{h} / 5, p_{T}^{ \pm}>10 \mathrm{GeV}$, ii) Jet rapidity for Tevatron (LHC): $|\eta|<2(2.5)$ iii) The angle between the missing transverse momentum and the muon direction: $\phi(\mu, \pm)>160^{\circ}$. 


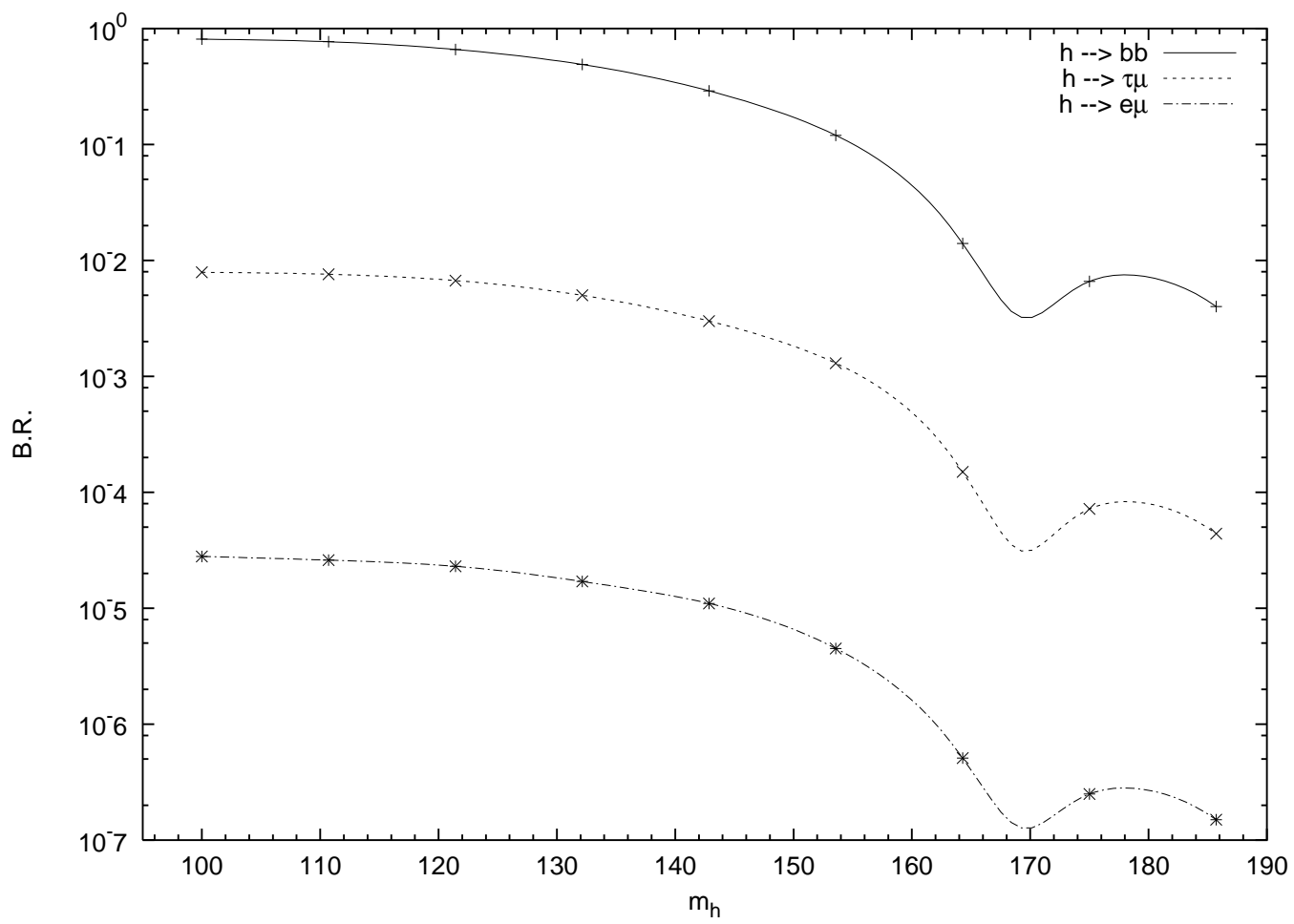

FIG. 1: Branching ratios of the Higgs boson, with $\kappa_{\tau \mu}=0.1$. Solid $(b \bar{b})$, Dashes $(\tau-\mu)$ and dot-dash $(e-\mu)$.

The resulting bounds on the LFV higgs couplings $\kappa_{\tau \mu}$ that can be obtained at Run-2 and LHC at $95 \%$ c.l., are shown in Fig. 2; one can see that it will be possible to test values of $\kappa_{\tau \mu}$ of order 1.2-1.5 (0.15-0.2) at Tevatron (LHC) with $4(100) \mathrm{fb}^{-1}$ for $m_{h}=110-130 \mathrm{GeV}$. In Fig. 2 we have also included the expected bound on $\kappa_{\tau \mu}$ at the very large hadron collider (VLHC), with c.m. energy of $40 \mathrm{TeV}$ and integrated luminosity of $1000 \mathrm{fb}^{-1}$, under the very crude assumption that signal and background can be scaled from LHC results; in this case the sensitivity extends up to values of $\kappa_{\tau \mu}=0.1$.

\section{CONCLUSIONS}

We have studied in this paper the conditions under which flavour violation can arise in scalar-fermion interactions, as a result of the mixing phenomena between the standard model and exotic fermions. This phenomena is then discussed within the specific context of a left-right model, which includes new fermions with mirror properties under the new gauge group $S U(2)_{R}$. Then we study phenomenological consequences of lepton flavor violation, focusing mainly in the Higgs sector of the model. Bounds on the parameters of the model are obtained from LFV processes; these results are then used to study the LFV Higgs decays $\left(h \rightarrow \tau l_{j}, l_{j}=e, \mu\right)$. We found that the LFV Higgs decays $h \rightarrow \tau \mu / \tau e$ can have large branching ratios, of order 0.01 . The signal $h \rightarrow \tau \mu$ has good chances to be detected at the coming stages of Tevatron Run-II. Further studies of the signal at Tevatron Run-2 is currently in progress [28], this time using the realistic detector simulation of CDF.

\section{Acknowledgments}

This work was partially supported by CONACyT, SNI and CIC-UMSNH in Mexico and COLCIENCIAS in Colombia.

[1] J. Maalampi and M. Roos, "Physics of mirror fermions," Phys. Rept. 186 (1990) 53. 


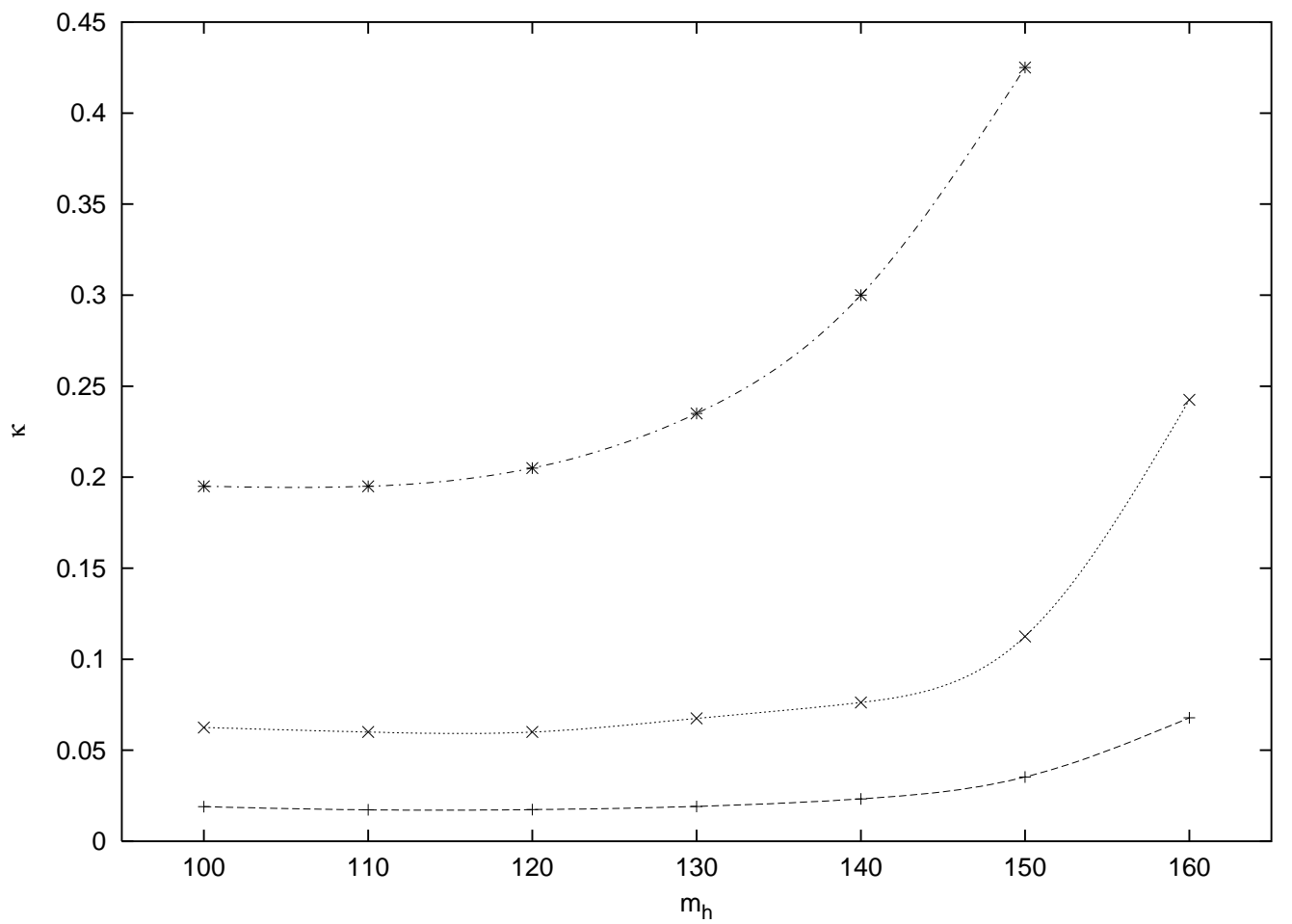

FIG. 2: Bounds on the LFV coupling $\kappa_{\tau \mu}$ that can be obtained at Tevatron Run-2 (dot-dashes), LHC (dots) and VLHC (dashes).

[2] Particle Data Group Collaboration, D. E. Groom et al., "Review of particle physics," Eur. Phys. J. C15 (2000) 1.

[3] V. E. Ceron, U. Cotti, J. L. Diaz-Cruz, and M. Maya, "Constraints on the parameters of the left-right mirror model," Phys. Rev. D57 (1998) 1934-1939, hep-ph/9705478.

[4] G. Triantaphyllou, "New physics with mirror particles," J. Phys. G26 (2000) 99-112, hep-ph/9811250.

[5] M. Lindner and G. Triantaphyllou, "Mirror families in electro-weak symmetry breaking," Phys. Lett. B430 (1998) 303-313, hep-ph/9803383.

[6] Z. G. Berezhiani and R. N. Mohapatra, "Reconciling present neutrino puzzles: Sterile neutrinos as mirror neutrinos," Phys. Rev. D52 (1995) 6607-6611, hep-ph/9505385.

[7] R. Foot and R. R. Volkas, "Neutrino physics and the mirror world: How exact parity symmetry explains the solar neutrino deficit, the atmospheric neutrino anomaly and the lsnd experiment," Phys. Rev. D52 (1995) 6595-6606, hep-ph/9505359.

[8] F. del Aguila and J. A. Aguilar-Saavedra, "Multilepton production via top flavour-changing neutral couplings at the cern lhc," Nucl. Phys. B576 (2000) 56-84, hep-ph/9909222.

[9] F. del Aguila, J. A. Aguilar-Saavedra, and L. Ametller, "Z-t and gamma-t production via top flavor-changing neutral couplings at the fermilab tevatron," Phys. Lett. B462 (1999) 310-318, hep-ph/9906462.

[10] J. L. Diaz-Cruz, R. Martinez, M. A. Perez, and A. Rosado, "Flavor changing radiative decay of thf t quark," Phys. Rev. D41 (1990) 891-894.

[11] J. L. Diaz-Cruz, M. A. Perez, G. Tavares-Velasco, and J. J. Toscano, "Testing flavor-changing neutral currents in the rare top quark decays $t \rightarrow c$ v(i) v(j)," Phys. Rev. D60 (1999) 115014, hep-ph/9903299.

[12] J. L. Diaz-Cruz and J. J. Toscano, "Probing lepton flavour violation with the higgs boson decays $h \rightarrow l_{i}^{+} l_{j}^{-}$" Phys. Rev. D62 (2000) 116005, hep-ph/9910233.

[13] P. Langacker and D. London, "Mixing between ordinary and exotic fermions," Phys. Rev. D38 (1988) 886.

[14] U. Cotti and A. Zepeda, "Model independent analysis of the simultaneous mixing of gauge bosons and mixing of fermions," Phys. Rev. D55 (1997) 2998-3005, hep-ph/9611442.

[15] E. Nardi, "Z', new fermions and flavor changing processes. constraints on $\mathrm{E}_{6}$ models from $\mu \rightarrow$ eee," Phys. Rev. D48 (1993) 1240-1247, hep-ph/9209223.

[16] T. D. Lee and C. N. Yang, "Question of parity conservation in weak interactions," Phys. Rev. 104 (1956) $254-258$.

[17] S. M. Barr, D. Chang, and G. Senjanovic, "Strong CP problem and parity," Phys. Rev. Lett. 67 (1991) $2765-2768$.

[18] K. S. Babu and R. N. Mohapatra, "A solution to the strong CP problem without an axion," Phys. Rev. D41 (1990) 1286.

[19] B. W. Lee and R. E. Shrock, "Natural suppression of symmetry violation in gauge theories: Muon - lepton and electron lepton number nonconservation," Phys. Rev. D16 (1977) 1444.

[20] G. Altarelli, L. Baulieu, N. Cabibbo, L. Maiani, and R. Petronzio, "Muon number nonconserving processes in gauge theories of weak interactions," Nucl. Phys. B125 (1977) 285. 
[21] L. N. Chang. D. Ng, and J. N. Ng, "Phenomenological consequences of singlet neutrinos," Phys. Rev. D50 (1994) 45894601, hep-ph/9402259.

[22] J. Bernabeu, E. Nardi, and D. Tommasini, "mu-e conversion in nuclei and Z' physics," Nucl. Phys. B409 (1993) 69-86, hep-ph/9306251.

[23] D. Ng and J. N. Ng, "Can mu-e conversion in nuclei be a good probe for lepton number violating higgs couplings?," Phys. Lett. B320 (1994) 181-185, hep-ph/9308352.

[24] M. Carena et al., "Report of the tevatron higgs working group," hep-ph/0010338.

[25] M. Sher, "Scalar mediated fcnc at the first muon collider," Phys. Lett. B487 (2000) 151-154, hep-ph/0006159

[26] T. Han and D. Marfatia, "h -i, mu tau at hadron colliders," Phys. Rev. Lett. 86 (2001) 1442-1445, hep-ph/0008141.

[27] A. Pilaftsis, "Lepton flavor nonconservation in h0 decays," Phys. Lett. B285 (1992) 68-74.

[28] U. Cotti, J. L. Diaz-Cruz, C. Pagliarone, and E. Vataga, "(work in progress),".

[29] We shall identify the light fermions as the SM ones, while the heavy ones $\left(\psi_{h}\right)$ will consider the sector beyond the SM, although this is not necessarily true, as a consequence of the fermion mixing phenomena. 